\title{
Regulation and Activity Evaluation of Secondary Metabolism of an Oyster Symbiotic Fungus Schizophyllum sp. YS-08
}

\author{
Huannan Wang, Maocai Yan, Mengdi Liang, Xiujian Wei, Zhao Shang, Zhen Zhang* \\ School of Pharmacy, Jining Medical University, Rizhao, China \\ Email address: \\ wanghuannan0322@126.com (Huannan Wang), yanmaocai@126.com (Maocai Yan), 2209321090@qq.com (Mengdi Liang), \\ 1061437922@qq.com (Xiujian Wei), 1259439883@qq.com (Zhao Shang), zhangzhen_1029@126.com (Zhen Zhang) \\ ${ }^{*}$ Corresponding author
}

\section{To cite this article:}

Huannan Wang, Maocai Yan, Mengdi Liang, Xiujian Wei, Zhao Shang, Zhen Zhang. Regulation and Activity Evaluation of Secondary Metabolism of an Oyster Symbiotic Fungus Schizophyllum sp. YS-08. International Journal of Microbiology and Biotechnology. Vol. 6, No. 3, 2021, pp. 78-85. doi: 10.11648/j.ijmb.20210603.13

Received: July 15, 2021; Accepted: July 29, 2021; Published: August 9, 2021

\begin{abstract}
Marine microorganisms are the important resources for natural drug discovery. However, most of genes are usually in silent and could not express under the condition of traditional culture, which limits the discovery of lead compounds. In the present research, marine fungus Schizophyllum sp. YS-08 was selected as the research object, which was from Yellow Sea of China, to activate its silent genes by changing the medium of nutrients and environmental conditions. The crude extracts from metabolites of marine fungi were analyzed by HPLC, and their antioxidant activity and acetylcholinesterase (AChE) inhibitory activity were studied by DPPH radical-scavenging and Ellman's method, respectively. The results indicated that metabolic pathway of fungus was regulated effectively in different culture conditions, especially in the normal sea water medium with peptone. The antioxidant activity and AChE inhibitory activity increased significantly in comparison with wild strain and increase of the quantity of chemical constituents were even more. It is also observed that the strains showed relatively slow growth in the high salinity situation, while this adversely environmental condition could promote and produce more active metabolites. Furthermore, 6 mutant strains were obtained under salt stress and were identified. Interestingly, we found that all mutant strains had potency toward antioxidant and AChE inhibitory activity. Among them, YS-08-2 was the most potent, scavenging more than 56.02\% towards DPPH free radicals, and YS-08-1, YS-08-4 and YS-08-5 exhibited more than 40\% inhibition against AChE. HPLC analysis of extracts showed the metabolites of most fungi were more abundant after regulation of culture conditions.
\end{abstract}

Keywords: Symbiotic Fungi, OSMAC Strategy, Secondary Metabolites, Antioxidant Activity, AChE Inhibitory Activity

\section{Introduction}

Secondary metabolites of marine microorganisms provide the most important sources of lead compounds in drug discovery. Because of the special environment for the growth of microorganisms, their secondary metabolites are usually very special and these compounds have high structural complexity and diversity [1-3]. However, currently, problems also existed in marine microorganisms such as few species, high repetition rate, single metabolites, etc, thus the development and utilization of marine natural products were limited under laboratory conditions [4-6]. Recently, research has found that there were still many silent genes existing in biosynthetic pathway of microorganisms and the activation of silenced genes again could enhance metabolites production or synthesis of novel compounds, which may provide new opportunity to the development of marine microorganism drugs [7-12].

One strain many compounds (OSMAC) strategy has been proposed recently, which become a convenient and effective method to investigate and explore marine microorganism natural products. The strategy is to activate the expression of silent genes in microorganisms by the change the composition of culture medium or culture conditions, so as to 
enhance the ability of microorganisms to synthesize secondary metabolites and then obtain more novel bioactive compounds [14-16]. At present a large number of researches indicated that OSMAC strategy has been successfully applied to induction and optimizition of secondary metabolites of marine microorganisms, especially fungi, because this method is simple, convenient and practicable [17-24]. In the present work, symbiotic fungus (Schizophyllum sp. YS-08) in wild oysters from Yellow Sea of China was used as the research object. To activate its silent metabolic pathway, the culture conditions and fermentation conditions were optimized by changing the medium of nutrients and environment. Moreover, the crude extracts from metabolites of endophytic fungi were analyzed by HPLC, and then their antioxidant activity and AChE inhibitory activity were evaluated by DPPH radical scavenging assay and Ellman's method, respectively. The purpose of this study is to provide a theoretical basis for the development of secondary metabolites of marine fungi.

\section{Materials and Methods}

\subsection{Materials}

Vitamin B1, Vitamin B2, Vitamin B3, Vitamin B5, Vitamin B6, Inositol, 4-aminobenzoic acid, Vitamin $\mathrm{H}, \mathrm{FeSO}_{4} \cdot 7 \mathrm{H}_{2} \mathrm{O}$, 5,5'-Dithiobis-(2-nitrobenzoic acid) (DTNB), Acetylthiocholine iodide (ATCI), Acetyl cholinesterase (AChE), $\quad \mathrm{NaH}_{2} \mathrm{PO}_{4} \cdot 2 \mathrm{H}_{2} \mathrm{O}, \quad$ 1,1-diphenyl-2-picrylhydrazyl
(DPPH), Ascorbic Acid, Glucose were purchased from Shanghai Yuanye Bio-Technology Co., Ltd (Shanghai, China). Peptone and Agar powder were from Beijing Laboratory Biology Technology Co., Ltd (Beijing, China). MNIQ-10 Column Fungi Genomic DNA Purification Kit was purchased from Sangon Biotech (Shanghai) Co., Ltd (Shanghai, China). All other chemical agents were from Sinopharm Chemical Reagent Co., Ltd (Shanghai, China).

\subsection{Fungal Materials}

Schizophyllum sp. YS-08 was isolated from wild oysters in Yellow Sea of China (longitude 119.45, latitude 35.42) and stocked at School of Pharmacy, Jining Medical University, Rizhao, China.

\subsection{Regulation of Secondary Metabolism of Oyster Symbiotic Fungus YS-08}

The effects on the secondary metabolism of fungus YS-08 with different growth factors were investigated. Oyster symbiotic fungus YS-08 was cultured in potato dextrose agar (PDA) medium, supplemented with peptone, multi-vitamins or $\mathrm{Fe}^{2+}$, respectively, as described in Table 1. The morphology and color of symbiotic fungi were observed after 5-7 days incubated at $28^{\circ} \mathrm{C}$ [25]. Next, we studied the effect on the secondary metabolism of fungus YS-08 with different salinities. The fungus YS-08 was incubated in the medium with different concentrations of sea salt (as described in Table 2), and cultured for 5-7 days.

Table 1. The fungus medium with different growth factors (seawater, $100 \mathrm{~mL}$ ).

\begin{tabular}{|c|c|c|c|c|c|}
\hline No. & Glucose (g) & Agar (g) & Peptone (g) & Multi-vitamins (mL) & $\mathrm{FeSO}_{4} \cdot 7 \mathrm{H}_{2} \mathrm{O}(\mathrm{mg})$ \\
\hline $1-1$ & 2 & 1.5 & - & - & - \\
\hline $1-2$ & 2 & 1.5 & - & 1 & - \\
\hline $1-3$ & 2 & 1.5 & - & - & 5 \\
\hline $1-4$ & 2 & 1.5 & - & 1 & 5 \\
\hline $1-5$ & 2 & 1.5 & 1 & - & - \\
\hline $1-6$ & 2 & 1.5 & 1 & 1 & - \\
\hline $1-7$ & 2 & 1.5 & 1 & - & 5 \\
\hline $1-9$ & 4 & 1.5 & - & 1 & - \\
\hline $1-10$ & 4 & 1.5 & - & - & 5 \\
\hline
\end{tabular}

Table 2. The fungus medium with different salinities (seawater, $100 \mathrm{~mL}$ ).

\begin{tabular}{|c|c|c|c|c|c|c|}
\hline No. & Glucose (g) & Agar (g) & Peptone (g) & Multi-vitamins (mL) & $\mathrm{FeSO}_{4.7} \cdot \mathrm{H}_{2} \mathrm{O}(\mathrm{mg})$ & Sea salt (g) \\
\hline $2-1-1 X$ & 2 & 1.5 & - & - & - & 3 \\
\hline $2-1-2 X$ & 2 & 1.5 & - & - & - & 6 \\
\hline $2-2-1 X$ & 2 & 1.5 & - & 1 & - & 3 \\
\hline $2-2-2 X$ & 2 & 1.5 & - & 1 & - & 6 \\
\hline $2-3-1 X$ & 2 & 1.5 & - & - & 5 & 3 \\
\hline $2-3-2 X$ & 2 & 1.5 & - & - & 5 & 6 \\
\hline $2-4-1 X$ & 2 & 1.5 & 1 & - & - & 3 \\
\hline
\end{tabular}

\subsection{Stability Study of Mutant Strains}

The oyster symbiotic fungi YS-08 was cultured in PDA medium with different growth factors (Table 1) or different salinities (Table 2). The mutant strains were isolated by the streak plate method and cultured in PDA medium. After the cultivation of three generations, they were very steady and hereditary, indicating that the mutant strains had a good genetic stability.

\subsection{Identification of Mutant Strains}

The mutant strains were inoculated to the potato dextrose 
broth (PDB) medium for spores growing, and the culture was shaken for $5 \sim 7 \mathrm{~d}$ at $150 \mathrm{r} / \mathrm{min}$. Then the spores were collected and ground to no particles. The genomic DNA was extracted from the spores with MNIQ-10 column fungi genomic DNA purification kit and was further amplified by PCR with fungal universal primers of ITS4 and ITS5. After amplification, agarose gel electrophoresis of the PCR products was preformed to make sure the products were the wanted fragments. At the same time, the verified products were submitted to Genewiz Corporation to confirm the sequencing. After the sequencing BLAST in the NCBI database, the phylogenetic tree was constructed based on Neighbor-Joining methods using the MEGA 7.0 software [26].

\subsection{Liquid Fermentation}

All the fungi were propagated in the potato-dextrose broth (PDB) medium and incubated at $28^{\circ} \mathrm{C}$ for $7-10 \mathrm{~d}$. Then the precipitate was removed by centrifugation, and the clear supernatant extract was extracted for 3 times with ethyl acetate. The crude extract was obtained after concentrating and stored at $4^{\circ} \mathrm{C}$.

\subsection{HPLC Analysis of Metabolites}

The crude extract was weighed and dissolved in methanol at concentration of $10 \mathrm{mg} / \mathrm{mL}$. The fingerprints of metabolites of symbiotic fungi were determined by high performance liquid chromatography (HPLC, Shimadzu), whose detector is DAD and whose mobile phase was methanol-water solution. HPLC method with gradient elution: 5\%-100\% methanol (0-40 min), $100 \%$ methanol (40-50 $\mathrm{min}), 100 \%-5 \%$ methanol (50-60 $\min )$.

\subsection{Bioactivity Screening for Secondary Metabolite}

The antioxidant activity of each crude extract was determined by using DPPH free radical scavenging method in vitro. A stock solution $(10 \mathrm{mg} / \mathrm{mL})$ of crude extract was prepared in DMSO. To a solution of $100 \mu \mathrm{L}$ DPPH $(0.4$ mmol/L) was added $2 \mu \mathrm{L}$ of crude extract, and then $98 \mu \mathrm{L} 75 \%$ ethanol was added in a 96-well plate in sextuplicate, and $2 \mu \mathrm{L}$ of $\mathrm{Vc}$ was added into positive control group, and $0.2 \mu \mathrm{L}$ of DMSO was added into the control group. After $30 \mathrm{~min}$ of incubation in the dark at room temperature, the absorbance was recorded at $517 \mathrm{~nm}$ on a microplate reader of multi-wavelength measurement system [27]. The DPPH free radical scavenging activity $(\%)$ was calculated by the formula $\left(\mathrm{A}_{0}-\left(\mathrm{A}_{\mathrm{i}}-\mathrm{A}_{\mathrm{i}}{ }^{\prime}\right)\right) / \mathrm{A}_{0} \times 100 \%$, where $\mathrm{A}_{0}$ is the absorbance value of control group, $\mathrm{Ai}$ is the absorbance value of test group, and $\mathrm{A}_{\mathrm{i}}{ }^{\prime}$ is the absorbance value of the crude extract itself.

The inhibitory activity of AChE was carried out by Ellman's method. A volume of $25 \mu \mathrm{L}$ of AChE solution $(0.05 \mu / \mathrm{L}), 16 \mu \mathrm{L}$ of DTNB solution $(0.0667 \mathrm{mmol} / \mathrm{L})$ and $10 \mu \mathrm{L}$ of ATCI solution $(0.1 \mathrm{mmol} / \mathrm{L})$ were added to 213.6 $\mu \mathrm{L}$ of $\mathrm{PB}$ buffer solution $(\mathrm{pH}=8.0)$, and then added with $5.4 \mu \mathrm{L}$ of each crude extract $(10 \mathrm{mg} / \mathrm{mL})$. After incubation of $4 \mathrm{~min} 20 \mathrm{~s}$ at $37^{\circ} \mathrm{C}$, the reaction mixture was treated with $10 \mu \mathrm{L}$ of SDS solution $(4 \mu \mathrm{mol} / \mathrm{L})$. The absorbance value of the reaction mixture was recorded at $412 \mathrm{~nm}$ [28]. The AChE inhibitory activities (\%) was measured according to the formula $\left(\left(\mathrm{A}_{0}-\mathrm{A}_{1}\right)-\left(\mathrm{A}_{\mathrm{i}}-\mathrm{A}_{\mathrm{i}}{ }^{\prime}\right)\right) /\left(\mathrm{A}_{0}-\mathrm{A}_{1}\right) \times 100 \%$, where $\mathrm{A} 0$ is the absorbance value of control, $A_{1}$ is the absorbance value of suppression group, $A_{i}$ is the absorbance value of test group, and $\mathrm{A}_{\mathrm{i}}{ }^{\prime}$ is the absorbance value of the crude extract itself.

\section{Results}

\subsection{Morphological Analysis of Fungus YS-08 in Different Growth Conditions}

To make silence gene of oyster symbiotic fungus express again, and to study the chemical diversity and bioactivity of its secondary metabolites, fungus YS-08 was cultured in PDA medium with different growth factors or different salinities. The strain was analyzed by color and morphological observation. The results showed that there was very little difference of morphological characteristic of strain when different growth factors were added in PDA medium (Figure 1A). However, the strains grew more vigorously and mycelium was dense hairy coronal. The color of strain was obviously deepened when peptone or $\mathrm{Fe}^{2+}$ was added. Interestingly, the addition of sea salt in the medium resulted in the inhibition of growth of the fungus, even to the extent, it could led to changes in colony morphology and color (Figure $1 \mathrm{~B})$, especially in $2 \mathrm{X}$ salinity culture media. Therefore, although growth of fungus was inhibited under the adverse conditions of salt stress, this could results in the appearance of some new mutant strains.

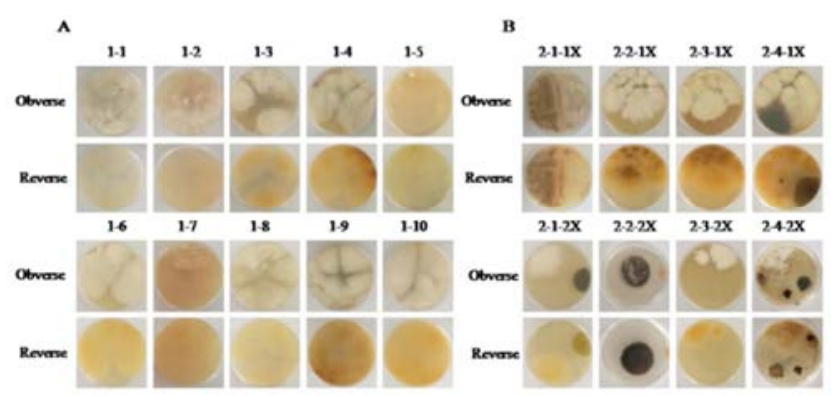

Figure 1. Morphological analysis on fungus YS-08 in different growth factors $(A)$ or different salinities $(B)$.

\subsection{HPLC Analysis of Crude Extract of Fungus YS-08 in Different Growth Conditions}

The fingerprint of main chemicals in crude extract of fungus YS-08 in different growth conditions was investigated by HPLC. As shown in Figure 2, the main peak ( $\left.t_{R} 33 \mathrm{~min}\right)$ of fingerprint of fungus YS-08 increased significantly when cultured in the PDB medium with multi-vitamins and iron ions (1-4), not single multi-vitamins (1-2) or iron ions (1-3). In addition, with the peptone addition, the number of peaks of fungus metabolites increased obviously (1-5, 1-6 and 1-7). Under the control of different salinities, the strains showed relatively slow growth speed and less aerial hyphae. Nevertheless, there were significant differences of the 
metabolites compared with cultured in normal medium. Furthermore, when the multi-vitamins $(2-2-1 X, 2-2-2 X)$ or peptone $(2-4-1 \mathrm{X}, 2-4-2 \mathrm{X})$ was added in the high salinity cultured medium, the metabolites were even more abundant.
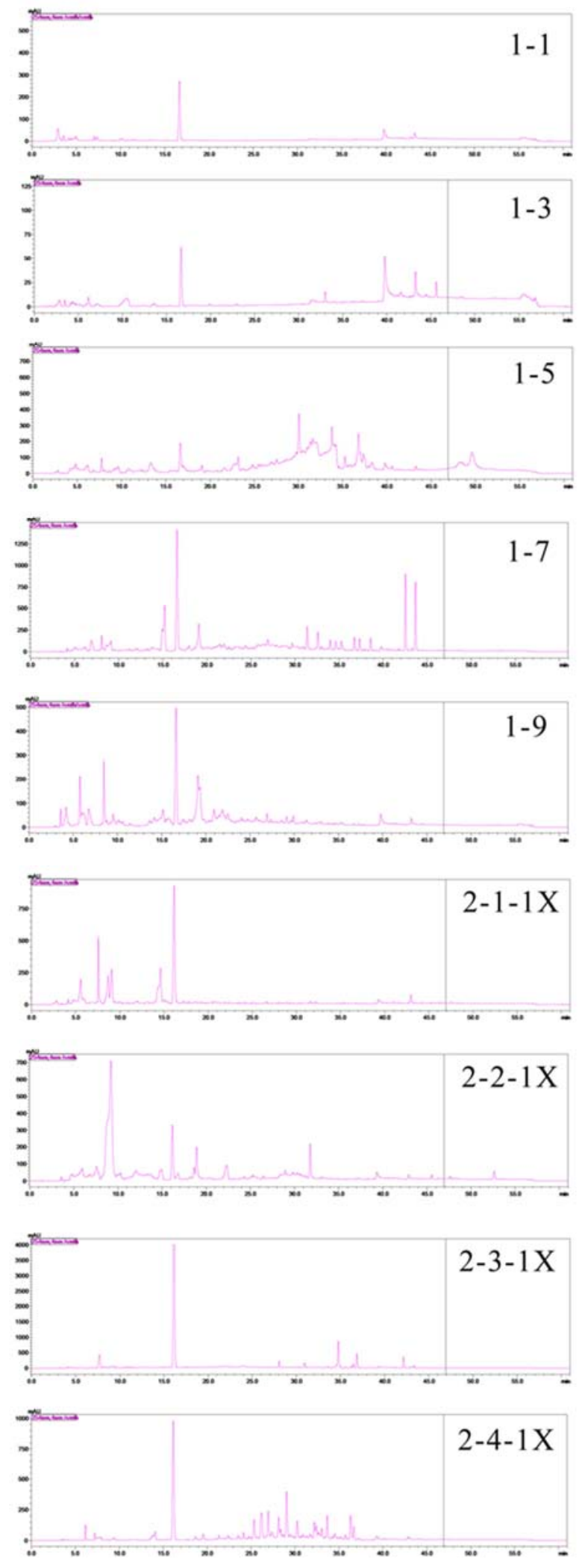

Figure 2. HPLC analysis on metabolites of fungus YS-08 in different growth conditions. gene of wild fungus [29].

Consequently, the concentration of sea salt was too high in the medium was unfavorable to fungal growth, but to stimulate the expression of metabolites by activating silence
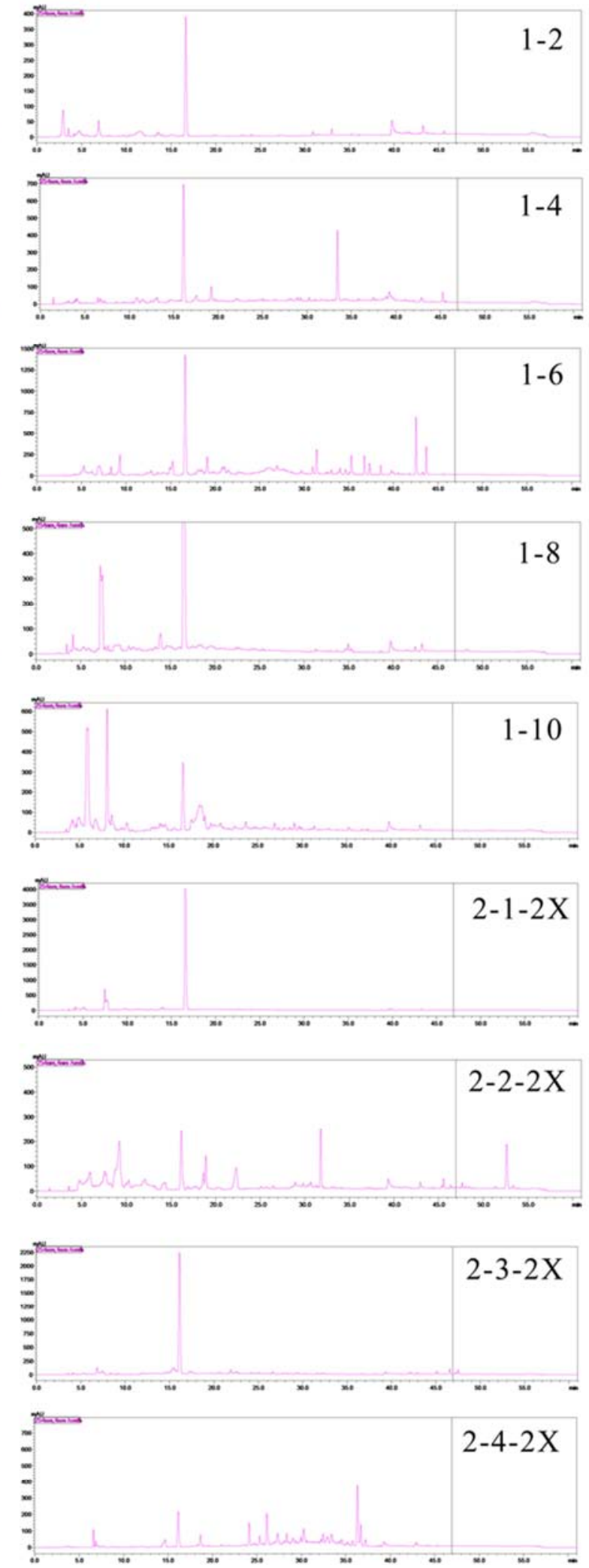


\subsection{Biological Activity of Crude Extract of Fungus YS-08 in Different Growth Conditions}

The antioxidant effects and AChE inhibitory activities of crude extract of fungus YS-08 in different growth conditions were tested by DPPH radical scavenging assay and Ellman assay in vitro. The results indicated that the antioxidant activity of metabolites was significantly increased in the dual

A

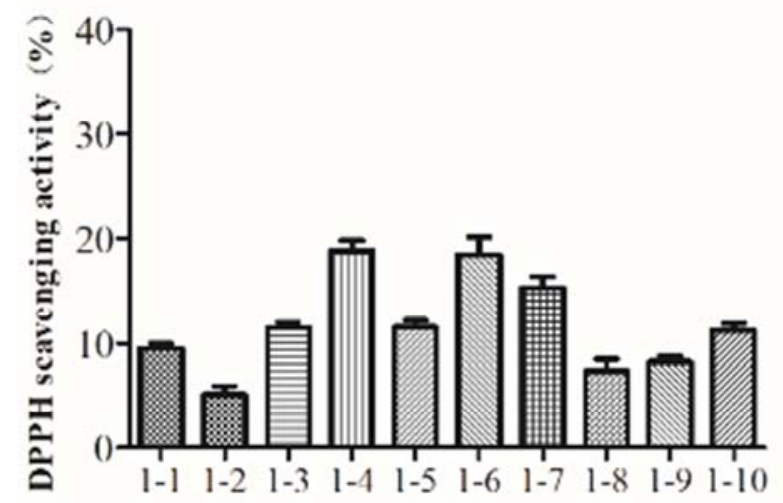

role of multi-vitamins and iron ion, and the scavenging rate was over $18.8 \%$ (Figure 3A). What is more, peptone in the culture medium has great effect on metabolites of endophytic fungus, and DPPH free radical scavenging rates were $11.52 \%$, $18.44 \%$ and $15.32 \%$ (Figure $3 \mathrm{~A}$ ), and the inhibitory rate of AChE were $19.04 \%, 31.95 \%$ and $27.54 \%$ (Figure 3B), respectively.

\section{B}

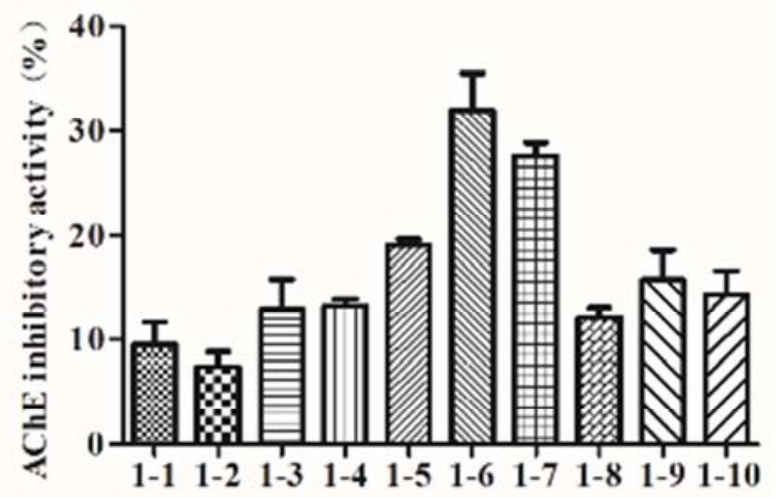

Figure 3. The influence of different growth factors on metabolites of endophytic fungus YS-08. A: DPPH free radical scavenging activity; B: AChE inhibitory activity.

Furthermore, the influence of different salinity conditions on metabolites of endophytic fungi was studied and the antioxidant activity and AChE inhibitory activity were investigated. Under $1 \mathrm{X}$ salinity conditions, $\mathrm{Fe}^{2+}$ or peptone was added into the medium could promote the activity of metabolites, and the scavenging rates of DPPH free radical were $24.15 \%$ and $19.15 \%$ (Figure $4 \mathrm{~A}$ ), and the inhibition rate

\section{A}

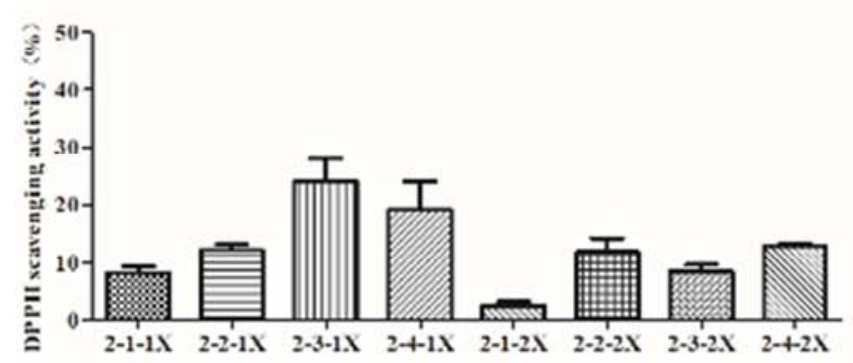

of AChE activity were $44.79 \%$ and $38.58 \%$ (Figure 4B). Under higher salinity stress $(2 \mathrm{X})$ the growth of fungi was restrained; however, the activities of metabolites increased, which might be regarded as appropriate salinity was beneficial to stimulate its silent metabolic pathway and produce new natural active products [30].

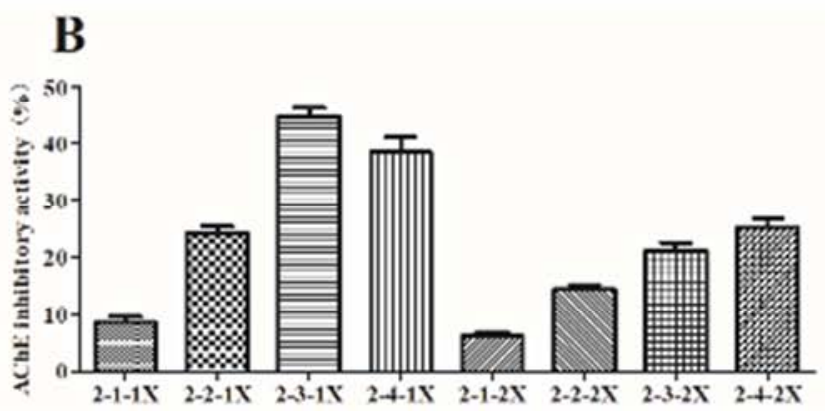

Figure 4. The influence of different salinity on metabolites of endophytic fungi YS-08. A: DPPH scavenging activity; B: AChE inhibitory activity.

\subsection{Identification of Mutant Strains}

Six species of mutant strains were isolated and purified when wild fungi (YS-08) was cultured in the above different salinity conditions. Subsequently, these mutant strains were preliminary identified through $16 \mathrm{~S}$ rDNA sequence analysis and locus in the development process (Figure 6) combined with their morphological features and culture characters (Figure 5). The results show that the species of the mutant strains were determined as Cladosporiumsphaerospermum (YS-08-1), Penicilliumrubens (YS-08-2, YS-08-4), Curvularialunata (YS-08-3), Aspergillusflavus (YS-08-5) and
Aspergillus sp. (YS-08-6), respectively.

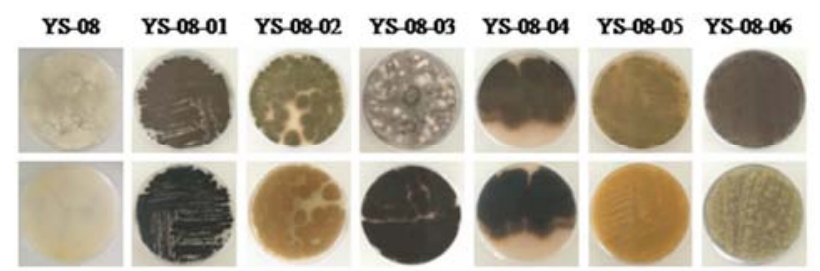

Figure 5. Morphological analysis of the mutant strains. 


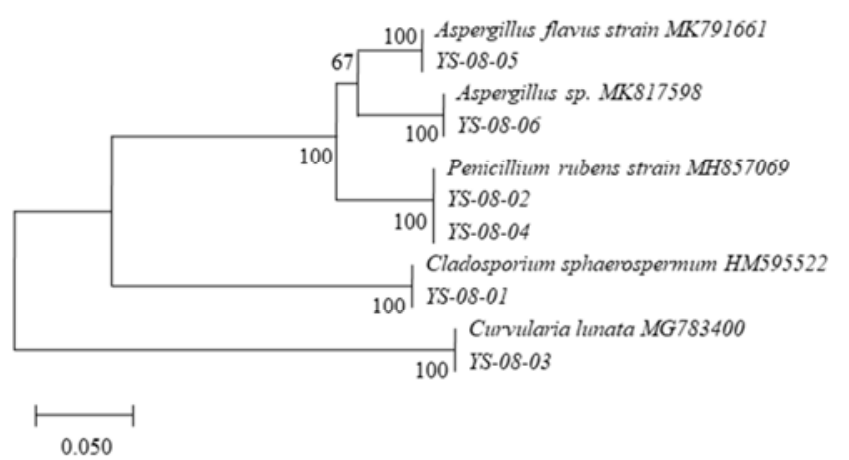

Figure 6. System development tree of the mutant strains.

A

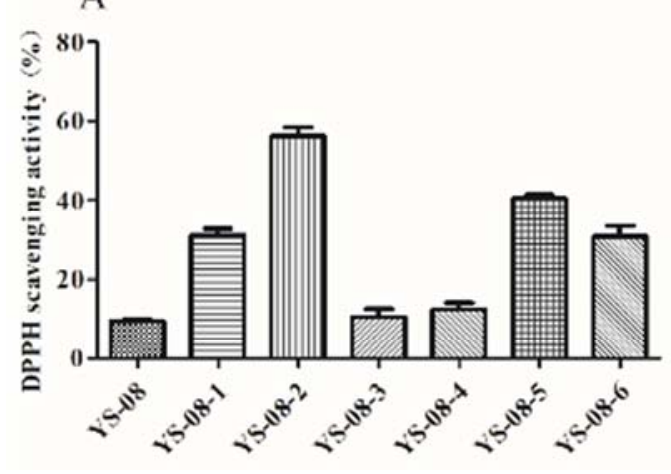

\subsection{Biological Activity of Metabolites of Mutant Strains}

The bioactivity of metabolites of 6 mutant strains was further studied. As shown in Figure 7, compared with wild strain YS-08, the antioxidant activity of 4 mutant strains (YS-08-1, YS-08-2, YS-08-5 and YS-08-6) were significantly increased, and the scavenging rate of DPPH radical were $31.14 \%, 56.02 \%, 40.48 \%$ and $31.16 \%$, respectively (Figure 7A). Inhibitory activity on $\mathrm{AChE}$ of all mutant strains were markedly enhanced, and the inhibitory rate of YS-08-1, YS-08-4 and YS-08-5 were up to $40.41 \%, 47.99 \%$ and $42.36 \%$, respectively.

\section{B}

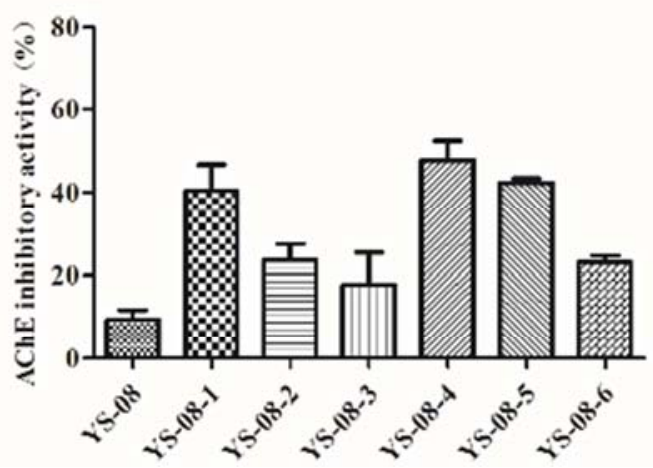

Figure 7. DPPH scavenging activity (A) and inhibitory activity on AChE (B) of metabolites of the mutant strains.

\subsection{HPLC Analysis on Metabolites of Mutant Strains}

The metabolites of 6 mutant strains were also analyzed by HPLC and we found that the marker peaks increased remarkably in comparison with YS-08 (Figure 2, Figure 8).
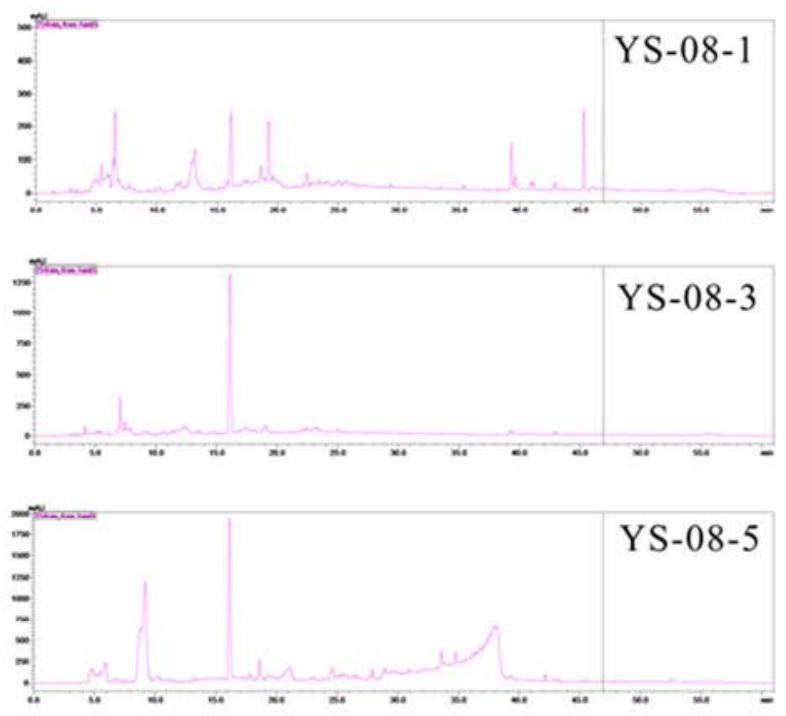

The results indicated that new secondary metabolites have been produced in mutant strains. According to the results of activity, structures of these compounds may be tightly related to the biological activities.
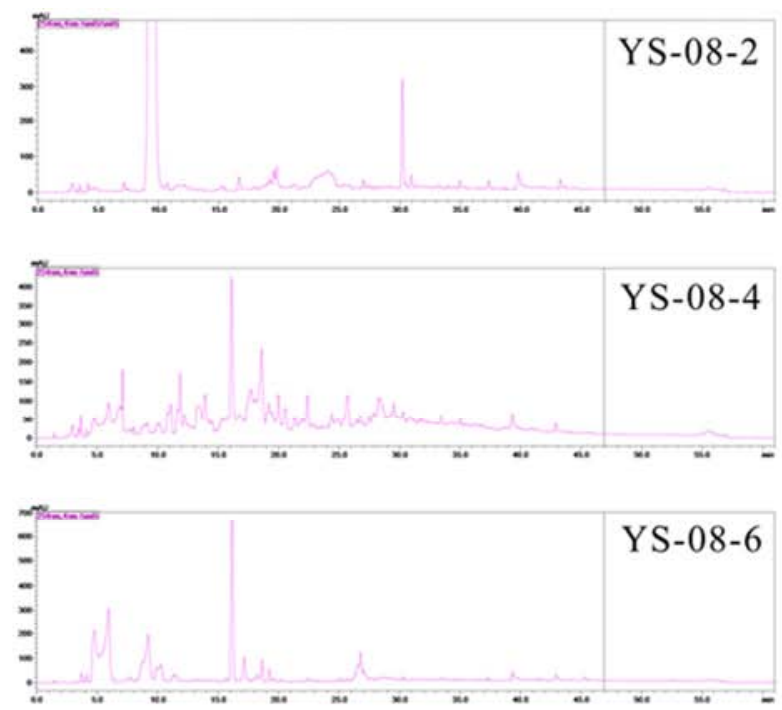

Figure 8. HPLC analysis on metabolites of the mutant strains.

\section{Discussion}

Chemical novelty and diversity of biological activities of the secondary metabolites from marine-derived microorganisms made a prominent contribution to the new lead compounds. Therefore, researching on the secondary metabolites of marine microorganisms have attracted the attentions of researchers [1, 31]. However, under the laboratory conditions, most of genes of marine microorganisms were usually in silent and could not express and resulted in failure to produce more novel structural 
compounds. The OSMAC strategy was first proposed by Zeeck [32] and have been widely used in the cultivation of microorganisms. This strategy can systematically change the growth parameters of microorganisms, and thus be beneficial for the diversity of their metabolites to some extent. Recent studies have shown that the change of fungal culture conditions can effectively regulate the production of secondary metabolites, and more compounds with novel structure and good biological activity could be obtained [19, 23]. For a better understanding of the impact of endophytic fungi on secondary metabolites under different culture conditions, oyster symbiotic fungi Schizophyllum sp. YS-08 was selected as the object in the present study.

The results showed that growth factor had significant effects on metabolic pathway of fungus, especially in the normal sea water salinity medium with peptone. In comparison with wild strain, the antioxidant activity and AChE inhibitory activity dramatically increased and the chemical constituents were even more abundant. Research has also found that the genes can be activated even under the adverse conditions, such as salt stress [29]. Consistently, the results of studies indicated that the bioactive metabolites produced by marine fungi are abundant in the high salinity situation despite the strains showed relatively slow growth speed. Furthermore, regulation of metabolites of fungi was more significant by adding $\mathrm{Fe}^{2+}$ or peptone to the previous salt stress condition. Meanwhile, 6 mutant strains were obtained under salt stress. Interestingly, we found that all mutant strains had potency toward antioxidant and AChE inhibitory activity. Among them, the DPPH free radical scavenging rates of YS-08-1, YS-08-2, YS-08-5, YS-08-6 were $31.14 \%$, 56.02\%, $40.48 \%$ and $31.16 \%$, and AChE inhibition rate of YS- $08-1$, YS-08-4 and YS-08-5 were $40.41 \%, 47.99 \%$ and $42.36 \%$. Analysis of extracts by HPLC showed the metabolites of most strains were more abundant after regulation of culture conditions.

\section{Conclusion}

The metabolic pathway of the oyster symbiotic fungi Schizophyllum sp. YS-08 was regulated effectively in different culture conditions. Under salt stress conditions, 6 mutant strains were obtained under salt stress and were identified to belong to Cladosporiumsphaerospermum YS-08-1, Penicilliumrubens YS-08-2 and YS-08-4, Curvularialunata YS-08-3, Aspergillusflavus YS-08-5, Aspergillus sp. YS-08-6, respectively. Furthermore, these strains could produce more active metabolites. Therefore, the OSMAC strategy has been successfully applied in the activation of silencing genes of oyster endophytic fungi. This study will supply a new approach to obtaining lead compounds with novel structures and diverse bioactivities in drug discovery.

\section{Conflicts of Interest}

There are no conflict of interest.

\section{Acknowledgements}

This study was supported by the Projects of Medical and Health Technology Development Program in Shandong Province (2019WS358), the Supporting Fund for Teachers' research of Jining Medical University (Nos. JYFC2018KJ026, JYFC2018KJ027), National Training Programs of Innovation and Entrepreneurship for Undergraduates (S202010443015) and the Jining Medical University Training Programs of Innovation and Entrepreneurship for Undergraduates (cx2020034).

\section{References}

[1] Li G., Lou H. X. (2018). Strategies to diversify natural products for drug discovery. Med Res Rev, 38, 1255-1294.

[2] Blunt J. W., Carroll A. R., Copp B. R., et al. (2018). Marine natural products. Nat Prod Rep, 35, 8-53.

[3] Carroll A. R., Copp B. R., Rohan A., et al. (2019). Marine natural products. Nat Prod Rep, 36, 122-173.

[4] Amend A., Burgaud G., Cunliffe M., et al. (2019). Fungi in the marine environment: open questions and unsolved problems. mBio, 10, e01189-18.

[5] Paranagama P. A., Wijeratne E. M. K., Gunatilaka A. L. (2007). Mncovering biosynthetic potential of plant-associated fungi: effect of culture conditions on metabolite production by paraphaeosphaeria quadriseptata and chaetomium chiversii. J Nat Prod, 70, 1939-1945.

[6] Pu X., Qu X. X., Chen F., et al. (2013). Camptothecin-producing endophytic fungus Trichoderma atroviride LY357: isolation, identification, and fermentation conditions optimization for camptothecin production. Appl Microbiol Biot, 97, 9365-9375.

[7] Wang W. X., Kusari S., Spiteller M. (2016). Unraveling the chemical interactions of fungal endophytes for exploitation as microbial factories. Fungal Appl Sustain Environ Biotech, 353-370.

[8] Yu P. W., Cho T. Y., Liou R. F., et al. (2017). Identification of the orsellinic acid synthase PKS63787 for the biosynthesis of antroquinonols in antrodia cinnamomea. Appl Microbiol Biot, $101,4701-4711$.

[9] Stroe M. C., Netzker T., Scherlach K., et al. (2020). Targeted induction of a silent fungal gene cluster encoding the bacteria-specific germination inhibitor fumigermin. eLife, 9, e52541.

[10] Yang Z. J., He J. Q., Wei X., et al. (2020). Exploration and genome mining of natural products from marine Streptomyces. Appl Microbiol Biot, 104, 67-76.

[11] Bauman K. D., Li J., Murata K., et al. (2019). Refactoring the cryptic streptophenazine biosynthetic gene cluster unites phenazine, polyketide, and nonribosomal peptide biochemistry. Cell Chem Biol, 26, 724-736.

[12] Kozlovsky A. G., Zhelifonova V. P., Antipova T. V., et al. (2010). The influence of medium compositiun on alkaloid biosynthesis by penicillium citrinum. Appl Biochem Micro, 46, 525-529. 
[13] Romano S., Jackson S. A., Patry S., et al. (2018). Extending the "One Strain Many Compounds" (OSMAC) principle to marine microorganisms. Mar Drugs, 16, 244.

[14] Pan R., Bai X. L., Chen J. W., et al. (2019). Exploring structural diversity of microbe secondary metabolites using OSMAC strategy: a literature review. Front Microbiol, 10, 294.

[15] Reen F. J., Romano S., Dobson A. D. W., et al. (2015). The sound of silence: activating silent biosynthetic gene clusters in marine microorganisms. Mar Drugs, 13, 4754-4783.

[16] Chai Y. J., Cui C. B., Li C. W., et al. (2012). Activation of the dormant secondary metabolite production by introducing gentamicin-resistance in a marine-derived penicillium purpurogenum G59. Mar Drugs, 10, 559-582.

[17] Lin Z. J., Zhu T. J., Wei H. J., et al. (2019). Spicochalasin A and new aspochalasins from the marine-derived fungus spicaria elegans. Eur J Org Chem, 18, 3045-3051.

[18] Abdelmohsen U. R., Cheng C., Viegelmann C., et al. (2014). Dereplication strategies for targeted isolation of new antitrypanosomal actinosporins $\mathrm{A}$ and $\mathrm{B}$ from a marine sponge associated-actinokineospora sp EG49. Mar Drugs, 12, $1220-1244$.

[19] Hewage R. T., Aree T., Mahidol C., et al. (2014). One strain-many compounds (OSMAC) method for production of polyketides, azaphilones, and an isochromanone using the endophytic fungus Dothideomycete sp. Phytochemistry, 108, 87-94.

[20] Zang Y., Gong Y. H., Gong J. J., et al. (2020). Fungal Polyketides with three distinctive ring skeletons from the fungus penicillium canescens uncovered by OSMAC and molecular networking strategies. J Org Chem, 85, 4973-4980.

[21] Almeida E. L., Kaur N., Jennings L. K., et al. (2019). Genome mining coupled with OSMAC-based cultivation reveal differential production of surugamide $\mathrm{A}$ by the marine sponge isolate streptomyces sp. SM17 when compared to its terrestrial relative S. albidoflavus J1074. Microorganisms, 7, 394.

[22] Zhao D. L., Han X. B., Wang D., et al. (2019). Bioactive 3-decalinoyltetramic acids derivatives from a marine-derived strain of the fungus fusarium equiseti D39. Front Microbiol 10, 1285 .

[23] Li W., Ding L. J., Wang N., et al. (2019). Isolation and characterization of two new metabolites from the sponge-derived fungus Aspergillus sp. LS34 by OSMAC approach. Mar Drugs, 17, 283.

[24] Zhang Z. Z., He X. Q., Che Q., et al. (2018). Sorbicillasins $\mathrm{A}-\mathrm{B}$ and scirpyrone $\mathrm{K}$ from a deep-sea-derived fungus, phialocephala sp FL30r. Mar Drugs, 16, 245.

[25] Wang H. N., Yang H., Gu Y. L., et al. (2019). Study on isolation, indentification and bioactivity of secondary metabolites from oysters symbiotic bacteria. J Liaocheng University, 32, 106-110.

[26] Saitou N., Nei M. (1987). The neighbor-joining method: a new method for reconstructing phylogenetic trees. Mol Biol Evol, 4, 406-425.

[27] Foti M. C. (2015). The use and abuse of the DPPH radical. J Agr Food Chem, 63, 8765.

[28] Zaki A. G., El-Shatoury E. H., Ahmed A. S., et al. (2019). Production and enhancement of the acetylcholinesterase inhibitor, huperzine A, from an endophytic Alternariabrassicae AGF041. Appl Microbiol Biot, 103, 5867-5878.

[29] Wang Y., Zheng J. K., Liu P. P., et al. (2011). Three new compounds from aspergill $\mu$ s terreus PT06-2 grown in a high salt medium. Mar Drugs, 9, 1368-1378.

[30] Huang J. J., Lu C. H., Qian X. M., et al. (2011). Effect of salinity on the growth, biological activity and secondary metabolites of some marine fungi. Acta Oceanol Sin, 30, 118-123.

[31] Marmann A., Aly A. H., Lin W. H., et al. (2014). Co-cultivation-a powerful emerging tool for enhancing the chemical diversity of microorganisms. Mar Drugs, 12, 1043-1065.

[32] Bode H. B., Bethe B., Hofs R., et al. (2002). Big effects from small changes: possible ways to explore nature's chemical diversity. Chembiochem, 3, 619-627. 